\title{
Management of endograft infection coupled with aortoesophageal fistula: Extra-anatomic aortic bypass and endograft explantation
}

\author{
Mariangela De Masi, MD, Philippe Amabile, MD, Laurence Bal, MD, and Philippe Piquet, MD, \\ Marseille, France
}

Thoracic endovascular aortic repair (TEVAR) is an accepted method of treating thoracic aortic aneurysms located on the descending thoracic aorta. ${ }^{1}$ Herein, we describe a case of a secondary aortoesophageal fistula (AEF), and its management.

\section{CLINICAL SUMMARY}

A man, aged 44 years, a tobacco user, was referred with thoracic back pain. The patient had a history of back pain for 7 months that had become unrelenting for the past 2 weeks. His blood test results revealed the following: hemoglobin, $100 \mathrm{~g} / \mathrm{L}$; leucocyte, $13.67 \times 10^{9} / \mathrm{L}$; and C-reactive protein, $181 \mathrm{mg} / \mathrm{L}$.

The computed tomographic angiography (CTA) revealed a bulky aneurysm (diameter, $65 \mathrm{~mm}$ ) of the descending thoracic aorta (Figure 1, $A$ ).

Emergency TEVAR was performed using a $26 \times 134-\mathrm{mm}$ Zenith endograft (Cook, Inc, Bloomington, Ind). The procedure was technically successful, and the patient was discharged from the hospital.

He was seen again 9 days later with back pain, asthenia, and sporadic bouts of slight fever; his blood test results revealed elevated inflammatory markers.

The CTA showed the presence of air bubbles in the excluded aneurysmal sac (Figure 1,B). An AEF was diagnosed. A gastrostomy was performed for enteral feeding, and intravenous antibiotics, piperacillin/tazobactam, were started. One week later, there was a clear improvement in pain and in the inflammatory syndrome. A CTA performed 1 month later showed an aortic aneurysm developing around the distal part of the endograft (Figure 1, $C$ ). Esophagogastroduodenoscopy was immediately followed by massive hematemesis. A second endograft was placed on the level of the developing aneurysm and successfully stopped the bleeding.

The persistence of the AEF and its complication, despite endovascular and medical treatment, motivate an open

\footnotetext{
From the Department of Vascular Surgery, Hôpital de la Timone, Marseille, France. Disclosures: Authors have nothing to disclose with regard to commercial support.

Received for publication April 18, 2013; accepted for publication April 24, 2013; available ahead of print June 17, 2013.

Address for reprints: Mariangela De Masi, MD, Department of Vascular Surgery,

Hôpital de la Timone, 264 rue St Pierre, 13385 Marseille Cedex 05, France

(E-mail: mdemasi@ap-hm.fr).

J Thorac Cardiovasc Surg 2013;146:e11-3

$0022-5223 / \$ 36.00$

Copyright (c) 2013 by The American Association for Thoracic Surgery

http://dx.doi.org/10.1016/j.jtcvs.2013.04.038
}

surgical procedure. An extra-anatomic bypass was fashioned between the ascending and the supraceliac aorta ("ventral aorta"). A 16-mm Dacron graft was anastomosed end to side to the supraceliac aorta and tunneled into the mediastinum through the diaphragm. The graft was carried along the lateral border of the right atrium, and anastomosed proximally to the right lateral aspect of the ascending aorta.

The second surgical stage was performed during the same procedure. The patient was placed in a lateral decubitus position. An anterolateral thoracotomy was performed, entering through the fifth intercostal space. After clamping, the aorta was opened longitudinally, and the 2 endografts were removed. The proximal and distal ends of the thoracic aorta were ligated with 4-0 polypropylene running sutures. The esophageal fistula was identified, debrided, and repaired with a simple suture. The chest and mediastinum were copiously irrigated and drained. Results of intraoperative cultures of the aortic wall were negative.

The patient had an uncomplicated postoperative recovery: a regular diet was resumed 2 weeks later, without swallowing difficulties, and the antibiotics were maintained for 10 days.

Three years after explantation of the endograft, the patient continues to do well (Figure 1, D).

\section{DISCUSSION}

Secondary AEF after TEVAR has been reported, with an incidence of $1.9 \%,{ }^{2}$ and can have disastrous outcomes. Several pathophysiologic mechanisms have been suggested for secondary AEF after TEVAR. The hypotheses include the following: (1) erosion of the relatively rigid stent-graft through the aorta directly into the esophagus, (2) necrosis of the esophageal wall because of continuous pressure of the self-expanding endoprosthesis, (3) ischemic esophageal necrosis because of stent-graft coverage of aortic side branches that feed the esophagus, and (4) infection of the stent-graft that eventually extends to the esophagus, eroding its wall. ${ }^{2}$

For our patient, potential ischemic necrosis of the esophageal wall because of coverage of the aortic side branches that feed the esophagus could be discussed. Endovascular thoracic aorta stenting for AEF might be useful as a temporary measure before definitive open surgery. However, it should only be considered as a stopgap measure to achieve short-term hemostasis until definitive treatment 


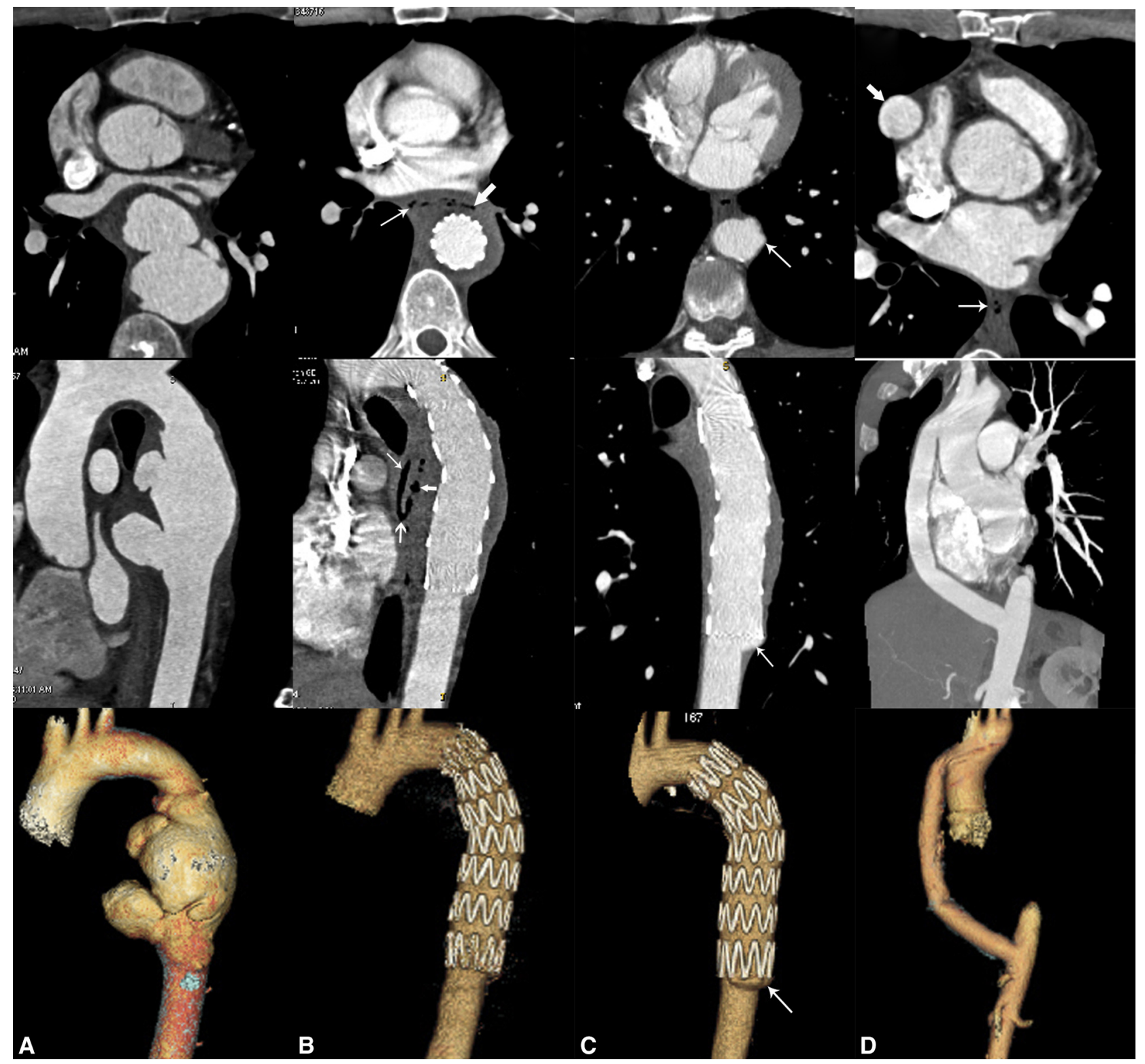

FIGURE 1. Vascular computed tomographic (CT) scan with axial native images (upper row), curved multiplanar reconstruction (middle row), and volumerendering reconstruction (lower row) acquired before any treatment (A), after the first and second endovascular procedures (B and C, respectively), and after surgical treatment (D). In A, a CT scan showed a huge aneurysm that compressed the esophagus and left atrium. In B, the CT scan showed air bubbles (large arrows) in the excluded aneurismal sac, esophageal lumen (thin arrow), and aortoesophageal fistula (open arrow). In C, an aortic aneurysm developed around the distal part of endograft (thin arrow). In D, the image shows 3 years after thoracic endograft explantation (thin arrow) and ventral aortic bypass (large arrow).

by conventional surgery within a few hours or days. ${ }^{3}$ This was the management method that we opted for.

Aortic graft replacement and esophageal fistula repair are the only definitive treatment for AEF. Aortic reconstruction can be performed in situ using synthetic grafts, cryopreserved arterial allografts, ${ }^{4}$ or an extra-anatomic bypass. We choose an extra-anatomic bypass arising from the ascending aorta (ventral aorta) to reduce the risk $^{5}$ by routing the graft away from the infected areas.
Treatment of the esophageal defect is mandatory. Direct suture is feasible only for small lesions that are detected early, with no significant mediastinitis, as in our case.

\section{CONCLUSIONS}

The treatment of secondary AEF after TEVAR can be achieved efficiently and durably by debridement and an extra-anatomic bypass. TEVAR remains a stopgap measure, 
although it is the best therapeutic strategy in an acute setting.

\section{References}

1. Dake MD. Endovascular stent-graft management of thoracic aortic diseases. Eur J Radiol. 2001;39:42-9.

2. Eggebrecht H, Mehta RH, Dechene A, Tsagakis K, Kühl H, Huptas S, et al. Aortoesophageal fistula after thoracic aortic stent-graft placement: a rare but catastrophic complication of a novel emerging technique. JACC Cardiovasc Interv. 2009;2:570-6.
3. Vallabhajosyula P, Komlo C, Wallen T, Szeto WY. Two-stage surgical strategy for aortoesophageal fistula: emergent thoracic endovascular aortic repair followed by definitive open aortic and esophageal reconstruction. J Thorac Cardiovasc Surg. 2012;144:1266-8.

4. Coselli JS, Crawford ES. Primary aortoesophageal fistula from aortic aneurysm successful surgical treatment by use of omental pedicle graft. J Vasc Surg. 1990;12:269-77.

5. Riesenman PJ, Farber MA. Management of a thoracic endograft infection through an ascending to descending extra-anatomic aortic bypass and endograft explantation. J Vasc Surg. 2010;51:207-9.

\title{
Uterine leiomyoma extension into right atrium: A case report
}

\author{
Hua-shan Xu, MD, ${ }^{\mathrm{a}}$ Khan Mohammed Firoj, MM, ${ }^{\mathrm{a}}$ Kamran Yunus Inamdar, MM, ${ }^{\mathrm{b}}$ and \\ Wen-zeng Zhao, MM, ${ }^{\mathrm{a}}$ Zhengzhou and Urumchi, China
}

Uterine leiomyoma is a common disease in women; however, intravenous leiomyomatosis of uterine origin extending via inferior vena cava (IVC) into the right side of the heart, known as intracardiac leiomyomatosis (ICL), is a rare condition. Because of rarity, ICL is occasionally misdiagnosed as a right atrial myxoma and thrombus.

\section{CASE REPORT}

A 42-year-old married premenopausal woman complaining of intermittent vaginal bleeding was referred to our department for an intracardiac mass. She had a 1-month history of progressive breathlessness, fatigue on exercise, and edema of her lower extremities. Her echocardiogram revealed a large mass in the right atrium, and the mass extended via the IVC far into the pelvic vein (Figure 1), which was confirmed on magnetic resonance imaging (Figure 2). Because of unfamiliarity to the disease, it was diagnosed as right atrial myxoma, combined with longstanding IVC thrombosis. Later, pelvic ultrasonography revealed multiple leiomyomas of the uterus, which made the attention toward the diagnosis of ICL.

The patient underwent a 2-stage surgery. In the first stage (transatrial tumor resection), the operation was performed under normal temperature with establishment of

\footnotetext{
From the Department of Cardiovascular Surgery, ${ }^{a}$ Institute of Clinical Medical Research of Universities Henan, The First Affiliated Hospital of Zhengzhou University, Zhengzhou, Henan, China; and the Cardiovascular Surgery Department, ${ }^{\text {b }}$ The First Affiliated Hospital of Xinjiang Medical University, Urumchi, Xin Jiang, China.

Disclosures: Authors have nothing to disclose with regard to commercial support.

Received for publication April 13, 2013; accepted for publication May 2, 2013; available ahead of print June 28, 2013.

Address for reprints: Hua-shan Xu, MD, Department of Cardiovascular Surgery, The First Affiliated Hospital of Zhengzhou University, Zhengzhou 450052, Henan,

China (E-mail: xhsay@ hotmail.com).

J Thorac Cardiovasc Surg 2013;146:e13-4

$0022-5223 / \$ 36.00$

Copyright (c) 2013 by The American Association for Thoracic Surgery

http://dx.doi.org/10.1016/j.jtcvs.2013.05.003
}

cardiopulmonary bypass (CPB) by draining the blood from a single superior vena cava and recirculating back through the aortic root. The tumor mass was having a tailed-like structure invaded into the IVC (Figure 3), which was pulled out gently through the right atrial incision. Immediately after pulling out the mass, the IVC cannula was advanced into the IVC. The surgical views revealed that the tumor within the vessel and heart had no stalk and had well-demarcated borders with the wall of vessel and heart (Figure 3). Subsequently, the pathological report also confirmed uterine smooth muscle origin. So, oral tamoxifen ( $10 \mathrm{mg} / \mathrm{d}$, twice a day for 3 months) was administered. The second-stage surgery (hysterectomy) was done 2 weeks later for removing multiple uterine leiomyomas. In the recent 3 years of follow-up, she had no symptoms.

\section{DISCUSSION}

ICL is exceedingly rare. Most patients are usually premenopausal (median age, 44 years). A review of the literature reveals that more than half of the women with ICL had

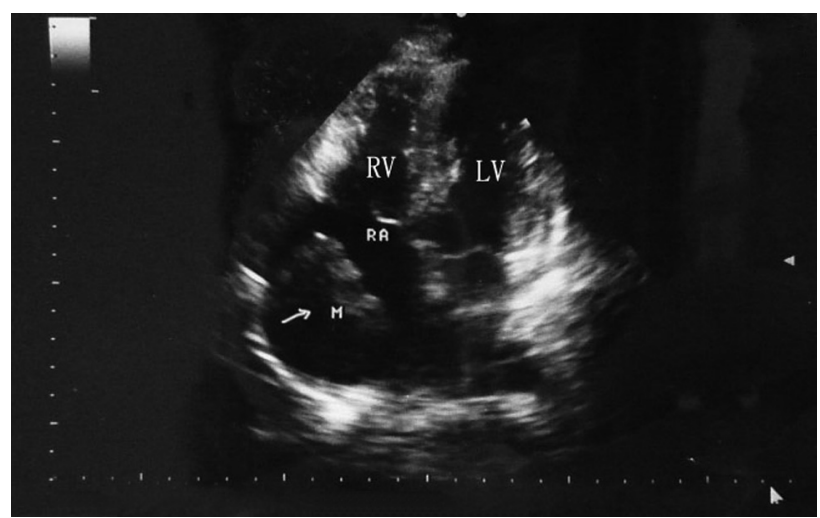

FIGURE 1. Apical 4-chamber view of echocardiography with a right atrial mass. $R V$, Right ventricle; $L V$, left ventricle; $R A$, right atrium; $M$, mass. 